\title{
Certification environnementale : la ruée vers le vert!
}

\author{
Caroline Boivin (Ph. D.) \\ Fabien Durif (Ph. D.) \\ Jean-Baptiste Drouillard (assistant de recherche) \\ Université de Sherbrooke
}

\section{INTRODUCTION}

Ces dernières années, en réponse aux changements des comportements de consommation, une multitude de marques vertes sont apparues sur le marché. Cependant, malgré la forte croissance de la présence et de la popularité des produits verts, de nombreux consommateurs restent encore sceptiques face à cette catégorie de produits.

Ces dernières années, en réponse aux changements des comportements de consommation, une multitude de marques vertes sont apparues sur le marché ${ }^{1}$. Cependant, malgré la forte croissance de la présence et de la popularité des produits verts, de nombreux consommateurs restent encore sceptiques face à cette catégorie de produits. Tout d'abord, certains sont réticents à acheter des produits verts ${ }^{2}$ à cause des risques qu'ils perçoivent en ce qui a trait à la qualité, au prix, au manque d'information ${ }^{3}$ ou à leur incapacité à délivrer les promesses environnementales ${ }^{4}$. Ensuite, une certaine confusion persiste au sujet de la compréhension des allégations environnementales des produits dits verts et beaucoup de consommateurs remettent en cause la crédibilité environnementale des entreprises. En effet, de nombreuses organisations profitent de la tendance verte pour «verdir» leurs produits et augmenter leurs ventes ${ }^{5}$, et les cas d'écoblanchiment se multiplient. En conséquence, de plus en plus de consommateurs sont préoccupés et recherchent des informations crédibles au sujet des attributs des produits verts.

Une des réponses apportées par les entreprises pour clarifier et valider les allégations environnementales est la certification environnementale. Celle-ci peut prendre plusieurs formes, dont les logos verts. La labellisation verte a d'ailleurs augmenté en popularité ces dernières années aussi bien dans le monde des affaires que dans les perceptions des consommateurs ${ }^{6}$. D'après le rapport de TerraChoice Environmental Marketing, de 2007 à 2009, la proportion d'écolabels est passée de 13,7 à 23,4 pour cent sur le marché nord-américain. Dans une étude de l'agence torontoise Bensimon Byrne en 2008, plus de 80 pour cent des consommateurs souhaiteraient que des normes soient imposées aux producteurs et qu'un étiquetage certifie et explique les termes utilisés sur les produits verts.

\section{De nombreuses organisations profitent de la tendance verte pour « verdir » leurs produits et augmenter leurs ventes, et les cas d'écoblanchiment se multiplient... Une des réponses apportées par les entreprises pour clarifier et valider les allégations environnementales est la certification environnementale.}

Ainsi, plusieurs catégories d'écolabels sont utilisées pour fournir une information sur les caractéristiques environnementales des produits. Toutefois, le problème majeur est qu'il y a une véritable prolifération des programmes de certification supportant les allégations des produits prétendus verts et cela participe à la confusion des consommateurs. Conséquemment, l'imprécision des étiquettes et des écolabels de nombreux produits retrouvés sur le marché fait en sorte que les consommateurs ont de la difficulté à définir ce qu'est véritablement un produit vert. Or, si les consommateurs perçoivent les écolabels comme une condition nécessaire, ils souhaitent surtout avoir accès à une information 
précise sur ces derniers ${ }^{7}$, car une majorité d'entre eux ne comprennent pas la signification exacte de certaines informations indiquées sur les écolabels. Acheter des produits verts s'avère donc de plus en plus complexe!

\section{Les écolabels ne sont pas toujours le fruit d'organismes officiels, puisque certains sont créés par les entreprises elles-mêmes. \\ L'utilisation de logos non officiels, mais qui peuvent être perçus comme tels par les consommateurs, devient de plus en plus courante.}

Une autre problématique majeure est que les écolabels ne sont pas toujours le fruit d'organismes officiels, puisque certains sont créés par les entreprises elles-mêmes. L'utilisation de logos non officiels, mais qui peuvent être perçus comme tels par les consommateurs, devient de plus en plus courante. Le dernier rapport de TerraChoice a clairement mis ce problème en évidence puisqu'il a ajouté un septième péché d'écoblanchiment, le péché d' «étiquette mensongère ». Une proportion de plus de 23 pour cent des produits verts étudiés entraient dans cette catégorie, soit des produits qui par des mots ou des images donnent l'impression d'être endossés par une tierce partie alors que ce n'est pas le cas.
Étant donné que le scepticisme des consommateurs sur les allégations environnementales des produits verts semble en grande partie le résultat d'une information inadéquate et difficile à comprendre sur les écolabels, il apparaît déterminant d'analyser le rôle et les pratiques en matière de certification environnementale. Le problème est que présentement la recherche sur les écolabels reste relativement faible et il s'avère difficile d'évaluer leur efficacité étant donné leur croissance exponentielle sur le marché. C'est pourquoi cet article propose un premier élément de réponse en étudiant les pratiques actuelles de certification verte dans les produits de grande consommation. Il expose un état des lieux de la certification verte telle que pratiquée par les fabricants en prenant comme contexte le secteur des produits d'entretien ménager sur le marché québécois. Pour atteindre cet objectif, un recensement de produits a été effectué dans 32 bannières. Il a permis d'identifier plus de 1205 produits arborant 52 écolabels différents et cinq types d'allégations environnementales. Les résultats dressent un portrait particulièrement préoccupant des stratégies de certification verte.

Un recensement de produits a été effectué dans 32 bannières. Il a permis d'identifier plus de 1205 produits arborant 52 écolabels différents et cinq types d'allégations environnementales.

\section{LE PHÉNOMÈNE DE LA CERTIFICATION ENVIRONNEMENTALE}

Trois stratégies sont possibles dans la mise en marché des produits verts : la présence d'aucune certification, la présence d'une certification sans explication et la présence d'une certification avec explication. "Ecolabelling is a voluntary method of environmental performance certifycation and labelling that is practised around the world. An ecolabel is a label which identifies overall environmental preference of a product or service within a specific product/service category based on life cycle considerations. » Concrètement, les logos verts sont ajoutés sur les produits pour certifier de leur conformité à des normes environnementales établies par des organismes responsables. La marque du produit est donc alliée à celle de l'organisme, et cette alliance est communiquée aux consommateurs via l'apposition du logo sur le produit, ce qui constitue une alliance de marques. Hormis les logos verts à proprement parler, les écolabels peuvent prendre plusieurs formes comme, par exemple, des symboles de sécurité environnementale ou des messages environnementaux. 


\section{Les écolabels sont avant tout un moyen de reconnaissance du produit vert pour le consommateur, mais aussi de communication des justifications environnementales du produit. En fait, ils informent le consommateur sur les caractéristiques tangibles du produit et lui donnent une certaine valeur.}

Il faut comprendre que les écolabels sont avant tout un moyen de reconnaissance du produit vert pour le consommateur ${ }^{6}$, mais aussi de communication des justifications environnementales du produit $^{7}$. En fait, ils informent le consommateur sur les caractéristiques tangibles du produit (ex. : qualité) et lui donnent une certaine valeur (ex. : prestige $)^{8}$. Ainsi, les labels supportent les organisations dans leur stratégie verte, car ils indiquent que le produit a des impacts réduits sur l'environnement et ils projettent une image verte renforçant la confiance des consommateurs et améliorant ainsi la réputation de l'organisation.

Plusieurs études ont relevé les effets bénéfiques de la présence d'un logo vert sur les produits en termes de diminution du risque perçu du consommateur, de volonté de payer plus cher et bien entendu d'augmentation des ventes ${ }^{8}$. Au niveau stratégique, les labels sont utilisés pour modifier le comportement d'achat des consommateurs. Ces résultats sont cohérents avec les conclusions de recherches sur les alliances de marques qui confirment que les attributs de la marque alliée peuvent se transférer au produit et contribuer à faciliter son acceptation sur le marché.

\section{LE RECENSEMENT DES PRODUITS D'ENTRETIEN MÉNAGER SUR LE MARCHÉ QUÉBÉCOIS}

Afin d'analyser les écolabels, nous avons opté pour une catégorie de produits dans laquelle les produits verts sont relativement nombreux : les produits d'entretien ménager. Un recensement a porté sur quatre catégories :

- détergents à vaisselle pour le lavage à la main;

- assouplisseurs liquides pour la lessive;

- savon liquide pour la lessive;

- nettoyants tout usage.

Le recensement a été réalisé au cours de l'été 2009 dans neuf types de détaillants de la région de Sherbrooke :

- magasins d'alimentation (6 bannières);

- pharmacies (5 bannières);

- magasins à grande surface (2 bannières);

- quincailleries (6 bannières);

- magasins à escompte (3 bannières);

- magasins spécialisés (6 bannières);

- boutique virtuelle (1 bannière);

- magasin entrepôt (1 bannière);

- dépanneurs (2 bannières).

Un total de 1205 produits et 133 marques ont été identifiés. Chaque produit a été examiné individuellement et la présence d'écolabels et d'allégations environnementales a été notée. Un total de 52 écolabels différents ont été identifiés. En outre, nous avons enquêté sur les autorités responsables des programmes de certification et avons recueilli des informations sur les écolabels identifiés à la fois sur internet et auprès des fabricants directement lorsque l'information n'était pas disponible.

Les écolabels ont été classifiés en quatre catégories :

- labels officiels : labels reconnus par les autorités publiques et sujets à l'évaluation d'une tierce partie (c'est-à-dire contrôlés par des organismes indépendants);

- labels dont les normes sont établies par une tierce partie, mais dont l'application n'est pas soumise à une vérification;

- labels maison : labels du secteur privé établissant leur propre système de contrôle et ayant des normes variables;

allégations vertes : autodéclarations environnementales présentes directement sur l'emballage des produits et qui ne sont pas vérifiées par des organismes indépendants (ex. : biodégradable, recyclable, non toxique, naturel). 


\section{LE PORTRAIT DE LA CERTIFICATION ENVIRONNEMENTALE SUR LE MARCHÉ DES PRODUITS D'ENTRETIEN MÉNAGER AU QUÉBEC}

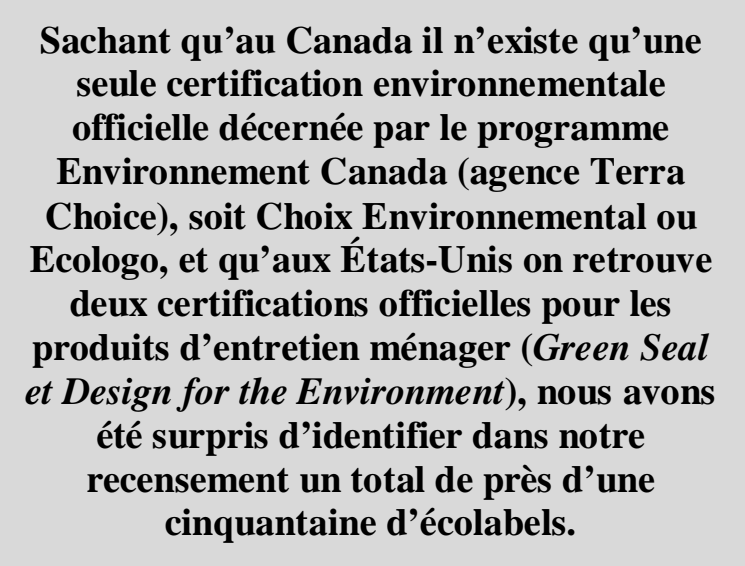

Le constat principal de notre recherche est que sur les 1205 produits d'entretien ménager du recensement, nous avons noté la présence de seulement trois écolabels officiels contre 49 écolabels non soumis à l'évaluation d'une tierce partie ou maison et cinq types d'allégations environnementales. Sachant qu'au Canada il n'existe qu'une seule certification environnementale officielle décernée par le programme Environnement Canada (agence Terra Choice), soit Choix Environnemental ou Ecologo, et qu'aux États-Unis on retrouve deux certifications officielles pour les produits d'entretien ménager (Green Seal et Design for the Environment), nous avons été surpris d'identifier dans notre recensement un total de près d'une cinquantaine d'écolabels. Le tableau 1 présente les écolabels officiels identifiés dans le recensement.

De manière plus précise, à l'été 2009, on retrouvait très peu de produits et de marques disposant d'écolabels officiels sur le marché des produits d'entretien ménager. En effet, moins de 10 pour cent des marques arboraient un écolabel officiel. Notons toutefois que cette proportion a assurément augmenté au cours des derniers mois, car des visites en magasin nous ont permis d'identifier plusieurs nouveaux produits apparus sur les tablettes sur lesquels des écolabels officiels sont apposés.

À l'été 2009, on retrouvait très peu de produits et de marques disposant d'écolabels officiels sur le marché des produits d'entretien ménager. En effet, moins de 10 pour cent des marques arboraient un écolabel officiel.

Tableau 1 : Écolabels officiels identifiés dans le recensement

\begin{tabular}{|l|c|}
\hline \multicolumn{1}{|c|}{ Écolabels } & Symboles visuels \\
\hline Programme Choix Environnemental & \\
Écologo & \\
Design for the environment &
\end{tabular}

Tel qu'indiqué au tableau 2, neuf différents types d'écolabels de la deuxième catégorie, c'est-à-dire les écolabels élaborés à partir de normes développées par des tierces parties mais qui ne sont pas soumis à une évaluation, ont été identifiés. Notons qu'à l'exception des labels « recyclables » et « haute efficacité » qui sont plus couramment utilisés, les écolabels selon les critères d'une tierce partie sont surtout présents dans une seule catégorie (produits nettoyants tout usage). Le tableau 2 présente les différents types d'écolabels et leurs déclinaisons qui peuvent différer d'un produit à l'autre. 


\section{Tableau 2 : Écolabels élaborés selon les critères d'une tierce partie identifiés dans le recensement}

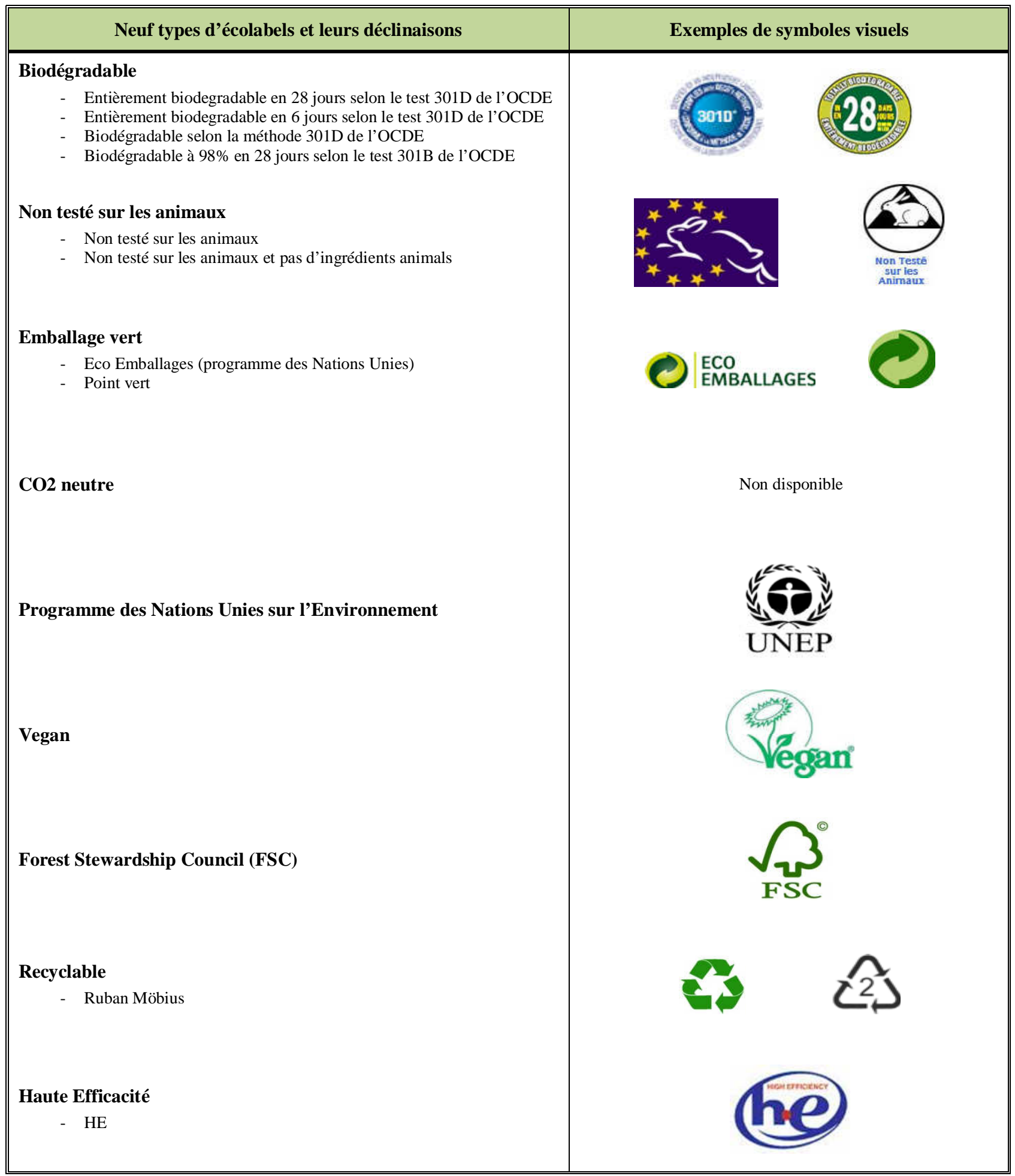


Tel qu'indiqué au tableau 3, sept types d'écolabels maison ont été identifiés, pour un total de 35 écolabels. Les écolabels maison sont présents dans toutes les catégories de produits d'entretien ménager. Ces écolabels se retrouvent évidemment uniquement sur des produits particuliers (ou une famille de produits de même marque), ce qui peut expliquer leur grand nombre.

\section{Tableau 3 : Écolabels maison identifiés dans le recensement}

\begin{tabular}{|c|c|}
\hline Sept types d'écolabels maison & Exemples de symboles visuels \\
\hline $\begin{array}{l}\text { Logos verts maison } \\
\text { - Éco LOGIQUE (Wal-Mart) } \\
\text { - } \text { Environnement vert (Essentiel) } \\
\text { - } \text { Nature Clean Earthy For } 30 \text { Years (Nature Clean) } \\
\text { - } \text { Respecte l'environnement (Fruits \& Passion) } \\
\text { - } \text { Formule biodégradable, emballage recyclable, concentré, moins de } \\
\text { - } \text { rebuts, aide à protéger l'environnement (Purex) } \\
\text { - } \text { Aucun produit chimique inutile (Palmolive) } \\
\text { - } \text { Eespect de la vie (L'Écolo) } \\
\text { - } \text { Bon pour la planète (Method) } \\
\text { - Liste verte / Greenlist (SC Johnson) } \\
\text { - } \text { Terre (Choix du Président) } \\
\text { - Eau (Choix du Président) } \\
\text { - Ingrédients naturels et d'origine végétale (Écover) } \\
\text { - Écologique/Rapidement biodégradable (Souris Verte) }\end{array}$ & 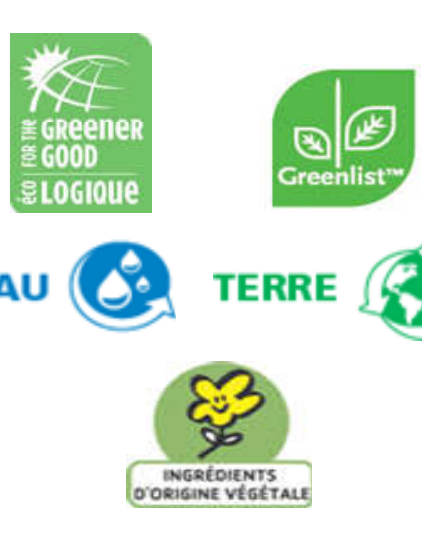 \\
\hline $\begin{array}{l}\text { Logos non toxiques maison } \\
\begin{aligned}- & \text { Bon pour les gens (Method) } \\
- & \text { Non toxique et écologique (Method) } \\
- & \text { Énergie (Choix du Président) }\end{aligned}\end{array}$ & ENER \\
\hline $\begin{array}{l}\text { Logos hypoallergéniques maison } \\
\text { - Peau sensible / hypoallergenique (Souris Verte) } \\
\text { - } \text { Hypoallergène / sans colorants (Purex) } \\
\text { - } \text { Aucun parfum lourd (Palmolive) } \\
\text { - Colorants non irritants (Palmolive) } \\
\text { - Avec désinfectant à l'huile de thym (Nature Clean) } \\
\text { - Extraits de parfums naturels (Purex) }\end{array}$ & Non disponibles \\
\hline $\begin{aligned} \text { Logos sans phosphates, sans chlorine, sans ammoniac maison } \\
-\quad \text { Sans ammoniac } \\
-\quad 100 \% \text { sans phosphates (Arctic Power) } \\
-\quad \text { Sans chlore, sans phosphates (Nature Clean) }\end{aligned}$ & Non disponibles \\
\hline $\begin{array}{l}\text { Logos sans enzymes maison } \\
\begin{aligned}- & 100 \% \text { Sans extraits fermentaires actifs (Lemieux) } \\
- & \text { Sans enzymes (Bionature) }\end{aligned}\end{array}$ & \\
\hline $\begin{array}{l}\text { Logos écotechnologiques maison } \\
-\quad 100 \% \text { Eco-Technologie (Lemieux) } \\
\text { - } \quad \text { Truly éprouvé (logo de Truly - marque maison de Zellers) } \\
-\quad \text { Le pouvoir nettoyant des agrumes (Bionature) }\end{array}$ & $\frac{100 \%}{30 \%}$ \\
\hline $\begin{array}{l}\text { Autres types de logos maison } \\
\begin{aligned} \text { - } & \text { SAFE for infant clothing } \\
- & \text { Sûr pour les fosses septiques } \\
- & \text { Non toxique pour la vie aquatique } \\
- & \text { Sans CFC }\end{aligned}\end{array}$ & 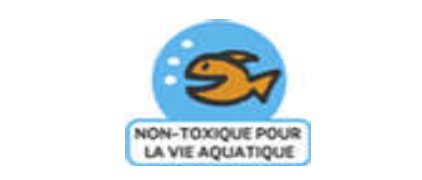 \\
\hline
\end{tabular}


Les allégations vertes ont été classifiées en cinq catégories tel qu'indiqué au tableau 4. Sans surprise, les allégations reliées à l'environnement sont les plus présentes sur le marché des produits d'entretien ménager (ex. : «respecte l'environnement »; « biodégradable »; « naturel »; «ph neutre »; «sans danger pour la vie aquatique, les humains, les animaux et les plantes »; « ne contient pas de produits dérivés du pétrole »; «connu pour une chimie sûre »).
Les allégations reliées à la santé se positionnent également comme une stratégie relativement importante utilisée par les entreprises (ex. : hypoallergénique, sans odeur/sans parfum, non cancérigène, non allergène, etc.). Par contre, les allégations reliées à la philosophie de l'entreprise n'ont été identifiées que sur l'emballage des produits d'une seule marque sur le marché. Le tableau 4 présente les allégations environnementales identifiées dans le recensement.

\section{Tableau 4 : Allégations environnementales identifiées dans le recensement}

\begin{tabular}{|c|c|}
\hline \multicolumn{2}{|c|}{ Allégations reliées à l'environnement } \\
\hline $\begin{array}{l}\text { - Respecte l'environnement } \\
\text { - Sans danger pour la vie aquatique, les humains, les } \\
\text { - } \text { animaux et les plantes } \\
\text { - } \text { Naturel } \\
\text { - Biodégradable } \\
\text { - Non toxique } \\
\text { - Ne contient pas de phosphates } \\
\text { - Ne contient pas d'ingrédients dérivés du pétrole } \\
\text { - Ne contient pas d'ammoniaque } \\
\text { - Ne contient pas de teintures/colorants }\end{array}$ & $\begin{array}{l}\text { - } \text { Certifié écologique } \\
\text { - Ne contient pas NTA/EDTA } \\
\text { - Reconnu pour une chimie plus sure } \\
\text { - Ne contient pas de javellisant } \\
\text { - } \mathrm{CO}_{2} \text { neutre } \\
\text { - Sans ethersulfate sodique de lauryle } \\
\text { - Sans agent de conservation } \\
\text { - Sans enzymes } \\
\text { - Sans alkylphénol } \\
\text { - Ne contribue pas à la formation d'algues bleues }\end{array}$ \\
\hline $\begin{array}{l}\text { Allégation reliées à la performance verte et non } \\
\text { verte } \\
\text { - Nettoyant biotechnologique } \\
\text { - } 2 x \text { plus concentré } \\
\text { - Testé en laboratoire } \\
\text { - Nettoyant écologique performant }\end{array}$ & $\begin{array}{l}\text { Allégations reliées à la santé } \\
\text { - Hyppoallergénique } \\
\text { - Ne contient pas de parfum } \\
\text { - Produit vegan } \\
\text { - Non-cancérigène } \\
\text { - Non-allergène } \\
\text { - Non-mutagène }\end{array}$ \\
\hline $\begin{array}{l}\text { Allégations reliées à la philosophie de l'entreprise } \\
\text { - Nous croyons que les produits ménagers devraient } \\
\text { nettoyer en profondeur tout naturellement, être faits } \\
\text { d'ingrédients de source végétale et minérale, } \\
\text { utiliser des ingrédients biodégradables, ne jamais } \\
\text { être testés sur des animaux. }\end{array}$ & $\begin{array}{l}\text { Allégations reliées à l'engagement social } \\
\text { - Une partie des profits sera remis à un organisme } \\
\text { environnemental reconnu. }\end{array}$ \\
\hline
\end{tabular}




\section{LES PRATIQUES ACTUELLES DE LA CERTIFICATION VERTE REMISE EN QUESTION}

\section{Le principal constat de notre étude est qu'il y a à la fois un nombre croissant et une qualité différente des écolabels créant ainsi une confusion de plus en plus importante aussi bien parmi les consommateurs que les professionnels.}

Le principal constat de notre étude est qu'il y a à la fois un nombre croissant et une qualité différente des écolabels créant ainsi une confusion de plus en plus importante aussi bien parmi les consommateurs que les professionnels. Dans son étude, l'organisme canadien Ecolabelling.org avait identifié 24 écolabels au Canada et en tout 78 écolabels en Amérique du Nord. Notre étude, uniquement sur les produits d'entretien ménager, a mis en avant un nombre encore plus important d'écolabels, soit 52, avec la présence de seulement trois écolabels officiels contre 49 écolabels non officiels et cinq types d'allégations environnementales, malgré le fait que deux écolabels officiels existent au Canada (Choix Environnemental et ÉcoLogo). L'un des points les plus importants est que moins de 10 pour cent des marques présentes sur le marché des produits d'entretien ménager arboraient une certification officielle. Ainsi, la quasi-totalité des produits d'entretien ménager dits verts sur le marché québécois sont endossés par des écolabels non officiels, la plupart du temps émanant des fabricants eux-mêmes. Outre le phénomène de prolifération d'écolabels non officiels, nous avons été surpris de relever un nombre extrêmement élevé d'allégations environnementales, soit des autodéclarations vertes des fabricants, directement sur les emballages des produits, augmentant en conséquence encore plus le niveau de confusion et la méfiance des consommateurs sur les prétentions vertes du produit.

La quasi-totalité des produits d'entretien ménager dits verts sur le marché québécois sont endossés par des écolabels non officiels, la plupart du temps émanant des fabricants eux-mêmes.

Figure 1: Impact de la certification environnementale

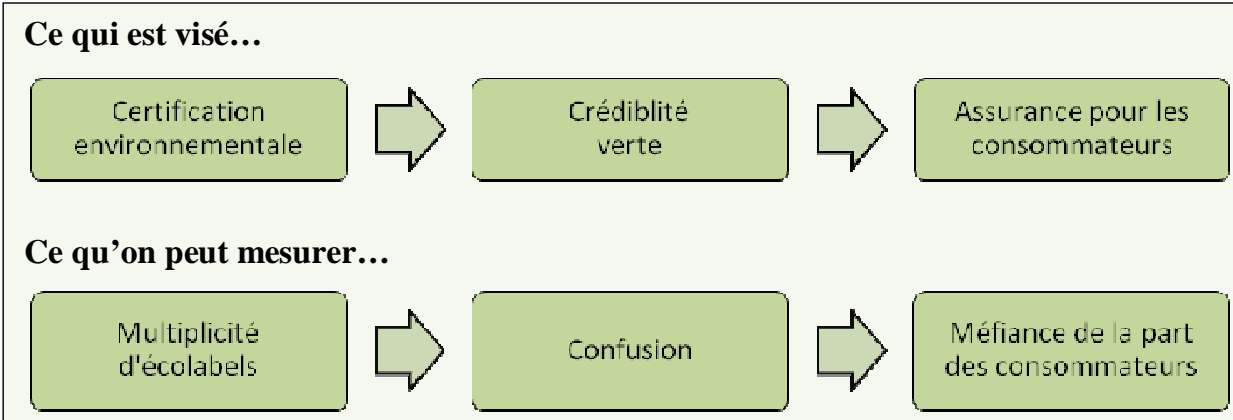

Il faut comprendre que la croissance et le manque d'harmonie dans la gestion des écolabels créent présentement une «barrière » psychologique pour les consommateurs. Les fabricants ont aujourd'hui tendance à exagérer les attributs verts de leurs produits par une utilisation excessive d'écolabels et d'allégations vertes sur les emballages ${ }^{7}$. Cela a comme résultat de développer des attitudes négatives des consommateurs envers les écolabels et finalement envers le produit lui-même. En fait, les consommateurs trouvent les allégations vertes plus crédibles si elles sont attribuées à des marques vertes et non à des marques traditionnelles ${ }^{3}$. 


\section{CONCLUSION}

\section{L'utilisation actuelle de la certification} environnementale permet seulement de rassurer la conscience des consommateurs sans véritablement les éduquer sur les impacts environnementaux réels de l'utilisation des produits.

Dans cette étude, nous avons noté que, dans la très grande majorité des cas, aucune information concernant les écolabels n'était disponible aussi bien sur l'emballage du produit que sur le site Web du fabricant. Or, une récente recherche en Australie a démontré que plus de 67,2 pour cent des consommateurs lisaient toujours les informations sur les écolabels ${ }^{7}$. Afin d'éviter toute confusion, il semble nécessaire que les fabricants disposent sur les emballages de leurs produits ou sur leurs sites Web des informations claires et précises sur les normes affiliées à l'obtention des écolabels et surtout sur l'organisme responsable du contrôle de la certification. L'utilisation actuelle de la certification environnementale permet seulement de rassurer la conscience des consommateurs sans véritablement les éduquer sur les impacts environnementaux réels de l'utilisation des produits'. En fait, la certification environnementale ne doit pas être considérée uniquement en tant que moyen de reconnaissance d'un produit vert pour les consommateurs (aspect marketing). Elle devrait être utilisée comme un véritable outil de politique environnementale pour promouvoir l'innovation verte et la concurrence entre les fabricants ${ }^{6}$. Par conséquent, il serait préférable pour les gouvernements d'établir une politique officielle de certification environnementale qui pourrait être adoptée par les organisations. Des travaux de recherche ont montré qu'un label endossé par une tierce partie officielle est l'élément le plus important pour les consommateurs, car il prouve que les attributs verts des produits sont contrôlés par des organismes officiels externes indépendants du fabricant ${ }^{1}$. Si une seule certification officielle verte était implantée et que la législation interdisait tout autre type d'allégations vertes non contrôlées, les entreprises offrant sur le marché des produits « réellement» verts auraient ainsi un meilleur retour sur investissement de leur engagement environnemental fort dans leurs processus. Par ailleurs, les entreprises qui «verdissent» superficiellement leurs produits pour améliorer leur image seraient plus facilement reconnaissables pour les consommateurs ${ }^{6}$. D' ailleurs, dans de nombreux pays, le nombre d'écolabels est limité et régi par un encadrement législatif, lequel a pour conséquence de diminuer la méfiance des consommateurs (ex. : seulement quatre écolabels officiels en Grande-Bretagne pour déterminer l'impact écologique d'un produit; «Eco Mark » au Japon; «Blue Angel » en Allemagne; ou «NF Environnement» en France).

Si une seule certification officielle verte était implantée et que la législation interdisait tout autre type d'allégations vertes non contrôlées, les entreprises offrant sur le marché des produits « réellement » verts auraient ainsi un meilleur retour sur investissement de leur engagement environnemental fort dans leurs processus.

\section{BIBLIOGRAPHIE}

${ }^{1}$ D'Souza, C., \& Taghian, M. (2005). "Green Advertising Effects on Attitude and Choice of Advertising Themes", Asia Pacific Journal of Marketing and Logistics, vol. 17, no 3, p. 51-66.

${ }^{2}$ Durif, F., Roy, J., Dubé, F., \& Lebrun, K. (2009). « Towards a better understanding of consumer's reluctance to buy green products: An exploratory study on perceived risks », Proceedings of the International Nonprofit and Social Marketing (INSM) Conference, Melbourne (Australia), July 14-15, 9 pages.

${ }^{3}$ Ginsberg, J., \& Bloom, P. (2004). "Choosing the Right Green-Marketing Strategy », MIT Sloan Management Review, vol. 46, p. 79-84.

${ }^{4}$ D'Souza, C., Taghian, M., \& Khosla, R. (2007). «Examination of Environmental Beliefs and its Impact on the Influence of Price, Quality and Demographic Characteristics with Respect to Green Purchase Intention », Journal of Targeting, Measurement and Analysis for Marketing, vol. 15, no 2, p. 69-78. 
${ }^{5}$ Peattie, K., \& Crane, A. (2005). «Green Marketing: Legend, Myth, Farce or Prophesy?», Qualitative Market Research: And International Journal, vol. 8, no 4, p. 357-370.

${ }^{6}$ Dosi, C., \& Moretto, M. (2001). « Is ecolabelling a Reliable Environmental Policy Measure?», Environ-ment and Resource Economics, vol. 18, no 1, p. 113-127.

${ }^{7}$ D'Souza, C., Taghian, M., \& Lamb, P. (2006). «An Empirical Study on the Influence of Environmental Labels on Consumers », Corporate Communications, vol. 11, no 2, p. 162-173.

${ }^{8}$ Sammer, K., \& Wüstenhagen, R. (2006). «The Influence of Eco-labelling on Consumer Behaviour- Results of a Discrete Choice Analysis for Washing Machines », Business Strategy and the Environment, vol. 15, p. 185-189.

${ }^{9}$ Collins-Chobanian, S. (2001). «A Proposal for Environmental Labels: Informing Consumers on the Real Costs of Consumption », Journal of Social Philosophy, vol. 32, no 3, p. 334-356. 